\title{
Existence and Iterative Algorithm of Solutions for a System of Generalized Nonlinear Mixed Variational-Like Inequalities
}

\author{
Yan-Mei Du \\ Department of Mathematics, Tianjin Polytechnic University, Tianjin 300387, China \\ Correspondence should be addressed to Yan-Mei Du, duyanmei@tjpu.edu.cn \\ Received 29 December 2011; Accepted 16 January 2012 \\ Academic Editor: Yonghong Yao \\ Copyright (C) 2012 Yan-Mei Du. This is an open access article distributed under the Creative \\ Commons Attribution License, which permits unrestricted use, distribution, and reproduction in \\ any medium, provided the original work is properly cited. \\ We introduce and study a system of generalized nonlinear mixed variational-like inequality \\ problems (SGNMVLIPs) in Banach spaces. The auxiliary principle technique is applied to study \\ the existence and iterative algorithm of solutions for the SGNMVLIP. First, the existence of \\ solutions of the auxiliary problems for the SGNMVLIP is shown. Second, an iterative algorithm for \\ solving the SGNMVLIP is constructed by using this existence result. Finally, not only the existence \\ of solutions of the SGNMVLIP is shown but also the convergence of iterative sequences generated \\ by the algorithm is also proven. The technique and results presented in this paper generalize and \\ unify the corresponding techniques and results given in the literature.
}

\section{Introduction and Preliminaries}

Variational inequality theory, which was introduced by Stampacchia [1] in 1964, is an important part of nonlinear analysis. Various kinds of iterative algorithms to solve the variational inequalities have been developed by many authors, see [2-8] and the references therein. Variational-like inequality introduced by Parida et al. [9] is an important generalization of the variational inequalities and has significant applications in nonconvex optimization. It is worth mentioning that the projection method cannot be extended for constructing iterative algorithms for variational-like inequalities. To overcome this drawback, one uses usually the auxiliary principle technique which deals with finding a suitble auxiliary problems for the original problem. Further, this auxiliary problem is used to construct an algorithm for solving the original problems. Glowinski et al. [10] introduced this technique and used it to study the existence of a solution of variational-like inequality. Later, many authors extended this technique to suggest and analyze a number of algorithms for solving various classes of variational inequalities (see [11-19]). 
Recently, the auxiliary principle technique was extended by Ding et al. [15] to study the existence and iterative algorithm of solutions of generalized strongly nonlinear mixed variational-like inequalities in Banach spaces. On the other hand, the auxiliary principle technique was also extended by Kazmi and Khan [16] who studied a system of generalized variational-like inequality problems in Hilbert spaces.

In this paper, we still extend the auxiliary principle technique to study a system of generalized nonlinear mixed variational-like inequality problems (SGNMVLIPs) in Banach spaces. At first, the existence of solutions of the auxiliary problems for the SGNMVLIP is shown. Next, an iterative algorithm for solving SGNMVLIP is constructed by using this existence and uniqueness result. Finally, we prove the existence of solutions of the SGNMVLIP and the convergence of the algorithm. These results improve and generalize many corresponding results given in [12,13,16-18].

Throughout the paper unless otherwise stated, let $I=\{1,2\}$ be an index set. For each $i \in I$, let $B_{i}$ be a real Banach spaces with norm $\|\cdot\|_{i}$, let $B_{i}^{*}$ be the topological dual space of $B_{i}$, and let $\langle\cdot, \cdot\rangle_{i}$ be the generalized duality pairing between $B_{i}$ and $B_{i}^{*}$. Let $F_{i}: B_{1} \times B_{2} \rightarrow B_{i}^{*}, \eta_{i}$ : $B_{i} \times B_{i} \rightarrow B_{i}$ be nonlinear mappings, then we consider the following system of generalized nonlinear mixed variational-like inequality problems (SGNMVLIPs): for given $\omega_{i}^{*} \in B_{i}^{*}$, find $(x, y) \in B_{1} \times B_{2}$ such that

$$
\begin{aligned}
& \left\langle F_{1}(x, y)-\omega_{1}^{*}, \eta_{1}\left(v_{1}, x\right)\right\rangle_{1}+b_{1}\left(x, v_{1}\right)-b_{1}(x, x) \geq 0 \quad \forall v_{1} \in B_{1} \\
& \left\langle F_{2}(x, y)-\omega_{2}^{*}, \eta_{2}\left(v_{2}, x\right)\right\rangle_{2}+b_{2}\left(y, v_{2}\right)-b_{2}(y, y) \geq 0 \quad \forall v_{2} \in B_{2}
\end{aligned}
$$

where for each $i \in I$, the bifunction $b_{i}: B_{i} \times B_{i} \rightarrow \mathbb{R}$ is a real-valued nondifferential function with the following properties:

(i) $b_{i}$ is linear in the first argument;

(ii) $b_{i}$ is convex in the second argument;

(iii) $b_{i}$ is bounded, that is, there exists a constant $\gamma_{i}>0$ such that

$$
b_{i}\left(u_{i}, v_{i}\right) \leq \gamma_{i}\left\|u_{i}\right\|_{i}\left\|v_{i}\right\|_{i}, \quad \forall u_{i}, v_{i} \in B_{i}
$$

(iv) $b_{i}\left(u_{i}, v_{i}\right)-b_{i}\left(u_{i}, w_{i}\right) \leq b_{i}\left(u_{i}, v_{i}-w_{i}\right)$, for all $u_{i}, v_{i}, w_{i} \in B_{i}$;

Remark 1.1 (see [15]). (1) It follows from property (i), for any $u_{i}, v_{i} \in B_{i}, b_{i}\left(-u_{i}, v_{i}\right)=$ $-b_{i}\left(u_{i}, v_{i}\right)$. By property (iii), we have $b_{i}\left(-u_{i}, v_{i}\right) \leq \gamma_{i}\left\|u_{i}\right\|_{i}\left\|v_{i}\right\|_{i}$, and hence

$$
\left|b_{i}\left(u_{i}, v_{i}\right)\right| \leq \gamma_{i}\left\|u_{i}\right\|_{i}\left\|v_{i}\right\|_{i}, \quad \forall u_{i}, v_{i} \in B_{i}
$$

This shows that for any $u_{i}, v_{i} \in B_{i}, b_{i}\left(u_{i}, 0\right)=b_{i}\left(0, v_{i}\right)=0$.

(2) It follows from properties (iii) and (iv), for any $u_{i}, v_{i}, w_{i} \in B_{i}$,

$$
\begin{aligned}
& b_{i}\left(u_{i}, v_{i}\right)-b_{i}\left(u_{i}, w_{i}\right) \leq \gamma_{i}\left\|u_{i}\right\|_{i}\left\|v_{i}-w_{i}\right\|_{i} \\
& b_{i}\left(u_{i}, w_{i}\right)-b_{i}\left(u_{i}, v_{i}\right) \leq \gamma_{i}\left\|u_{i}\right\|_{i}\left\|w_{i}-v_{i}\right\|_{i} .
\end{aligned}
$$


Therefore, we have

$$
\left|b_{i}\left(u_{i}, v_{i}\right)-b_{i}\left(u_{i}, w_{i}\right)\right| \leq \gamma_{i}\left\|u_{i}\right\|_{i}\left\|v_{i}-w_{i}\right\|_{i}
$$

This implies that $b_{i}$ is continuous with respect to the second argument.

\section{Some Special Cases}

(1) If for each $i \in I, B_{i}$ is a real Hilbert space and $\omega_{i}^{*}=0$, then the SGNMVLIP (1.1) and (1.2) reduce to the problems: find $(x, y) \in B_{1} \times B_{2}$ such that

$$
\begin{array}{ll}
\left\langle F_{1}(x, y), \eta_{1}\left(v_{1}, x\right)\right\rangle_{1}+b_{1}\left(x, v_{1}\right)-b_{1}(x, x) \geq 0 & \forall v_{1} \in B_{1} \\
\left\langle F_{2}(x, y), \eta_{2}\left(v_{2}, x\right)\right\rangle_{2}+b_{2}\left(y, v_{2}\right)-b_{2}(y, y) \geq 0 \quad \forall v_{2} \in B_{2}
\end{array}
$$

The problem (1.7) has been studied by Kazmi and Khan [16].

(2) If index set $I=\{1\}, B_{1}=B_{2}=B$ and $F_{1}(x, x)=F_{2}(x, x)=T x-A x$ for each $x \in B$, where $A, T: B \rightarrow B^{*}$ are two single-valued mappings, then the SGNMVLIP (1.1) and (1.2) reduce to the problem: find $x \in B$ such that

$$
\left\langle T x-A x-\omega^{*}, \eta(v, x)\right\rangle+b\left(x, v_{1}\right)-b(x, x) \geq 0 \quad \forall v \in B .
$$

The problem (1.8) with $\omega^{*}=0$ was introduced and studied by Ding [17] in reflexive Banach spaces.

In brief, for appropriate and suitable choice of the mappings $F_{1}, F_{2}, \eta_{1}, \eta_{2}, b_{1}, b_{2}$, and the linear continuous functionals $\omega_{1}^{*}$ and $\omega_{2}^{*}$, one can obtain a number of the known classes of variational inequalities as special cases from SGNMVLIP (1.1) and (1.2) (see [6-8]).

We need the following basic concepts, basic assumptions and basic results which will be used in the sequel.

Definition 1.2. Let $D$ be a nonempty subset of a Banach space $E$ with the dual space $E^{*}$. Let $g: D \rightarrow E^{*}$ and $\eta: D \times D \rightarrow E$ be two mappings, then

(i) $g$ is said to be $\eta$-strongly monotone if there exists a constant $\sigma>0$ such that

$$
\langle g(u)-g(v), \eta(u, v)\rangle \geq \sigma\|u-v\|^{2}, \quad \forall u, v \in D,
$$

(ii) $g$ is said to be Lipschitz continuous if there exists a constant $\mu>0$ such that

$$
\|g(u)-g(v)\| \leq \mu\|u-v\|, \quad \forall u, v \in D,
$$

(iii) $\eta$ is said to be Lipschitz continuous if there exists a constant $\delta>0$ such that

$$
\|\eta(u, v)\| \leq \delta\|u-v\|, \quad \forall u, v \in D
$$


Definition 1.3. Let $D$ be a nonempty subset of a Banach space $E$ with the dual space $E^{*}$. Let $F: D \times D \rightarrow E^{*}, \eta: D \times D \rightarrow E$ be two mappings, then $F$ is said to be

(i) $(\lambda, \xi)$-Lipschitz continuous if there exist constants $\lambda, \xi>0$ such that

$$
\left\|F\left(u_{1}, v_{1}\right)-F\left(u_{2}, v_{2}\right)\right\| \leq \lambda\left\|u_{1}-u_{2}\right\|+\xi\left\|v_{1}-v_{2}\right\|, \quad \forall u_{1}, v_{1}, u_{2}, v_{2} \in D,
$$

(ii) $\eta$-strongly monotone in the first argument if there exists a constant $\varepsilon_{1}>0$ such that

$$
\left\langle F\left(u_{1}, v\right)-F\left(u_{2}, v\right), \eta\left(u_{1}, u_{2}\right)\right\rangle \geq \varepsilon_{1}\left\|u_{1}-u_{2}\right\|^{2}, \quad \forall u_{1}, u_{2}, v \in D,
$$

(iii) $\eta$-strongly monotone in the second argument if there exists a constant $\varepsilon_{2}>0$ such that

$$
\left\langle F\left(u, v_{1}\right)-F\left(u, v_{2}\right), \eta\left(v_{1}, v_{2}\right)\right\rangle \geq \varepsilon_{2}\left\|v_{1}-v_{2}\right\|^{2}, \quad \forall v_{1}, v_{2}, u \in D
$$

Assumption 1.4. For each $i \in I$, the mapping $\eta_{i}: B_{i} \times B_{i} \rightarrow B_{i}$ satisfies the following conditions:

(1) $\eta_{i}(u, v)=\eta_{i}(u, z)+\eta_{i}(z, v)$, for all $u, v, z \in B_{i}$;

(2) $\eta_{i}$ is affine in the second argument;

(3) for each fixed $u \in B_{i}$, the function $v \mapsto \eta_{i}(u, v)$ is continuous from the weak topology to the weak topology.

Remark 1.5. It follows from Assumption 1.4 (1) that $\eta_{i}(u, v)=-\eta_{i}(v, u)$ and $\eta_{i}(u, u)=0$ for any $u, v \in B_{i}$. Moreover, we can prove that $\eta_{i}$ is also affine in the first argument by Assumption 1.4 (1) and (2).

Lemma 1.6 (see [20]). Let X be a nonempty close convex subset of a Hausdorff linear topological space $E$, and let $\phi, \psi: X \times X \rightarrow \mathbb{R}$ be mappings satisfying the following conditions:

(i) for each $(x, y) \in X \times X, \psi(x, y) \leq \phi(x, y)$ and $\psi(x, x) \geq 0$ for each $x \in X$;

(ii) for each $x \in X, \phi(x, y)$ is upper semicontinuous with respect to $y$;

(iii) for each $y \in X$, the set $\{x \in X: \psi(x, y)<0\}$ is convex;

(iv) there exists a nonempty compact set $\Omega \subset X$ and $x_{0} \in \Omega$ such that $\psi\left(x_{0}, y\right)<0$ for any $y \in X \backslash \Omega$.

Then, there exists an $\bar{y} \in \Omega$ such that $\phi(x, \bar{y}) \geq 0$ for any $x \in X$.

\section{Auxiliary Problems and Algorithm}

In this section, we introduce the auxiliary problems to study the SGNMVLIP (1.1) and (1.2), and we give an existence theorem for a solution of the auxiliary problems. By using the existence theorem, we construct the iterative algorithm for solving the SGNMVLIP (1.1) and (1.2). 
For each $i \in I$, let $g_{i}: B_{i} \rightarrow B_{i}^{*}$ be a single-valued mappings Given $\left(x_{1}, x_{2}\right) \in B_{1} \times B_{2}$, we consider the following problems $P\left(x_{1}, x_{2}\right)$ : find $\left(z_{1}, z_{2}\right) \in B_{1} \times B_{2}$ such that

$$
\begin{aligned}
& \left\langle g_{1}\left(z_{1}\right)-g_{1}\left(x_{1}\right)+\rho\left[F_{1}\left(x_{1}, x_{2}\right)-\omega_{1}^{*}\right], \eta_{1}\left(v_{1}, z_{1}\right)\right\rangle_{1}+\rho\left[b_{1}\left(x_{1}, v_{1}\right)-b_{1}\left(x_{1}, z_{1}\right)\right] \geq 0 \quad \forall v_{1} \in B_{1}, \\
& \left\langle g_{2}\left(z_{2}\right)-g_{2}\left(x_{2}\right)+\rho\left[F_{2}\left(x_{1}, x_{2}\right)-\omega_{2}^{*}\right], \eta_{2}\left(v_{2}, z_{2}\right)\right\rangle_{2}+\rho\left[b_{2}\left(x_{2}, v_{2}\right)-b_{2}\left(x_{2}, z_{2}\right)\right] \geq 0 \quad \forall v_{2} \in B_{2},
\end{aligned}
$$

where $\rho>0$ is a constant. The problems are called the auxiliary problems for SGNMVLIP (1.1) and (1.2).

Remark 2.1. If for each $i \in I, B_{i}$ is a real Hilbert spaces, $\omega_{i}^{*}=0$ and $g_{i}=I$ are the identity mappings on $B_{i}$, then the auxiliary problems reduce to Kazmi and Khan's auxiliary problems in [16].

Theorem 2.2. For each $i \in I$, let $\eta_{i}: B_{i} \times B_{i} \rightarrow B_{i}$ be Lipschtiz continuous with constant $\delta_{i}>0$, and let $g_{i}: B_{i} \rightarrow B_{i}^{*}$ be $\eta_{i}$-strongly monotone and Lipschtiz continuous with constant $\sigma_{i}>0$ and $\mu_{i}>0$, respectively. Let $b_{i}: B_{i} \times B_{i} \rightarrow \mathbb{R}$ satisfy the properties (i)-(iv). If Assumption 1.4 holds, then the auxiliary problems $P\left(x_{1}, x_{2}\right)$ have a unique solution.

Proof. For each $i \in I$, define the mappings $\phi_{i}, \psi_{i}: B_{i} \times B_{i} \rightarrow \mathbb{R}$ by

$$
\begin{aligned}
& \phi_{i}\left(v_{i}, z_{i}\right)=\left\langle g_{i}\left(v_{i}\right)-g_{i}\left(x_{i}\right)+\rho\left[F_{i}\left(x_{1}, x_{2}\right)-\omega_{i}^{*}\right], \eta_{i}\left(v_{i}, z_{i}\right)\right\rangle_{i}+\rho\left[b_{i}\left(x_{i}, v_{i}\right)-b_{i}\left(x_{i}, z_{i}\right)\right], \\
& \psi_{i}\left(v_{i}, z_{i}\right)=\left\langle g_{i}\left(z_{i}\right)-g_{i}\left(x_{i}\right)+\rho\left[F_{i}\left(x_{1}, x_{2}\right)-\omega_{i}^{*}\right], \eta_{i}\left(v_{i}, z_{i}\right)\right\rangle_{i}+\rho\left[b_{i}\left(x_{i}, v_{i}\right)-b_{i}\left(x_{i}, z_{i}\right)\right],
\end{aligned}
$$

respectively.

We claim that the mappings $\phi_{i}, \psi_{i}$ satisfy all conditions of Lemma 1.6 in the weak topology. Indeed, since $g_{i}$ is $\eta_{i}$-strongly monotone with constant $\sigma_{i}>0$ and Remark 1.5 , it is clear that $\phi_{i}$ and $\psi_{i}$ satisfy condition (i) of Lemma 1.6. Since the bifunction $b_{i}$ is convex in the second argument and $\eta_{i}$ is affine in the second argument, it follows from Assumption 1.4 (3) and Remark 1.1 (2) that $z_{i} \mapsto \phi_{i}\left(v_{i}, z_{i}\right)$ is weakly upper semicontinuous. By Assumption 1.4 (1) and (2), and the property (ii) of $b_{i}$, it is easy to prove that the set $\left\{v_{i} \in B_{i}: \psi_{i}\left(v_{i}, z_{i}\right)<0\right\}$ is convex, hence the conditions (ii) and (iii) of Lemma 1.6 hold.

Let $w_{i}=\sigma_{i}^{-1}\left[\delta_{i}\left\|g_{i}\left(x_{i}\right)-g_{i}(0)\right\|_{i}+\delta_{i} \rho\left\|F_{i}\left(x_{1}, x_{2}\right)-\omega_{i}^{*}\right\|_{i}+\rho \gamma_{i}\left\|x_{i}\right\|_{i}\right]$ and $K_{i}=\left\{z_{i} \in B_{i}\right.$ : $\left.\left\|z_{i}\right\|_{i} \leq w_{i}\right\}$, then $K_{i}$ is a weakly compact subset of $B_{i}$. For any $z_{i} \in B_{i} \backslash K_{i}$, take $v_{i_{0}}=0 \in$ $K_{i}$. From Assumption 1.4 (1), Remark 1.1 (1), Lipschitz continuity of $\eta_{i}$, and the $\eta_{i}$-strongly monotonicity of $g_{i}$, we have

$$
\begin{aligned}
\psi_{i}\left(v_{i_{0}}, z_{i}\right)= & \psi_{i}\left(0, z_{i}\right) \\
= & \left\langle g_{i}\left(z_{i}\right)-g_{i}\left(x_{i}\right)+\rho\left[F_{i}\left(x_{1}, x_{2}\right)-\omega_{i}^{*}\right], \eta_{i}\left(0, z_{i}\right)\right\rangle_{i}+\rho\left[b_{i}\left(x_{i}, 0\right)-b_{i}\left(x_{i}, z_{i}\right)\right] \\
= & -\left\langle g_{i}(0)-g_{i}\left(z_{i}\right), \eta_{i}\left(0, z_{i}\right)\right\rangle_{i}-\left\langle g_{i}\left(x_{i}\right)-g_{i}(0), \eta_{i}\left(0, z_{i}\right)\right\rangle_{i} \\
& +\rho\left\langle\left[F_{i}\left(x_{1}, x_{2}\right)-\omega_{i}^{*}\right], \eta_{i}\left(0, z_{i}\right)\right\rangle_{i}+\rho\left[b_{i}\left(x_{i}, 0\right)-b_{i}\left(x_{i}, z_{i}\right)\right] \\
\leq & -\sigma_{i}\left\|z_{i}\right\|_{i}^{2}+\delta_{i}\left\|g_{i}\left(x_{i}\right)-g_{i}(0)\right\|_{i}\left\|z_{i}\right\|_{i}+\delta_{i} \rho\left\|F_{i}\left(x_{1}, x_{2}\right)-\omega_{i}^{*}\right\|_{i}\left\|z_{i}\right\|_{i}+\rho \gamma_{i}\left\|x_{i}\right\|_{i}\left\|z_{i}\right\|_{i} \\
= & -\sigma_{i}\left\|z_{i}\right\|_{i}\left\{\left\|z_{i}\right\|_{i}-\sigma_{i}^{-1}\left[\delta_{i}\left\|g_{i}\left(x_{i}\right)-g_{i}(0)\right\|_{i}+\delta_{i} \rho\left\|F_{i}\left(x_{1}, x_{2}\right)-\omega_{i}^{*}\right\|_{i}+\rho \gamma_{i}\left\|x_{i}\right\|_{i}\right]\right\}<0 .
\end{aligned}
$$


Therefore, the condition (iv) of Lemma 1.6 holds. By Lemma 1.6 there exists an $z_{i}^{*} \in B_{i}$ such that $\phi_{i}\left(v_{i}, z_{i}^{*}\right) \geq 0$ for all $v_{i} \in B_{i}$, that is

$$
\left\langle g_{i}\left(v_{i}\right)-g_{i}\left(x_{i}\right)+\rho\left[F_{i}\left(x_{1}, x_{2}\right)-\omega_{i}^{*}\right], \eta_{i}\left(v_{i}, z_{i}^{*}\right)\right\rangle_{i}+\rho\left[b_{i}\left(x_{i}, v_{i}\right)-b_{i}\left(x_{i}, z_{i}^{*}\right)\right] \geq 0 \quad \forall v_{i} \in B_{i} .
$$

For arbitrary $t \in(0,1]$ and $v_{i} \in B_{i}$, let $y_{i, t}=t v_{i}+(1-t) z_{i}^{*}$. Replacing $v_{i}$ by $y_{i, t}$ in $(2.4)$ and utilizing Assumption 1.4 (1) and (2), Remark 1.5, and the property (ii) of $b_{i}$, we obtain

$$
\begin{aligned}
0 & \leq\left\langle g_{i}\left(y_{i, t}\right)-g_{i}\left(x_{i}\right)+\rho\left[F_{i}\left(x_{1}, x_{2}\right)-\omega_{i}^{*}\right], \eta_{i}\left(y_{i, t}, z_{i}^{*}\right)\right\rangle_{i}+\rho\left[b_{i}\left(x_{i}, y_{i, t}\right)-b_{i}\left(x_{i}, z_{i}^{*}\right)\right] \\
& =-\left\langle g_{i}\left(y_{i, t}\right)-g_{i}\left(x_{i}\right)+\rho\left[F_{i}\left(x_{1}, x_{2}\right)-\omega_{i}^{*}\right], \eta_{i}\left(z_{i}^{*}, y_{i, t}\right)\right\rangle_{i}+\rho\left[b_{i}\left(x_{i}, y_{i, t}\right)-b_{i}\left(x_{i}, z_{i}^{*}\right)\right] \\
& \leq-t\left\langle g_{i}\left(y_{i, t}\right)-g_{i}\left(x_{i}\right)+\rho\left[F_{i}\left(x_{1}, x_{2}\right)-\omega_{i}^{*}\right], \eta_{i}\left(z_{i}^{*}, v_{i}\right)\right\rangle_{i}+\rho t\left[b_{i}\left(x_{i}, v_{i}\right)-b_{i}\left(x_{i}, z_{i}^{*}\right)\right] .
\end{aligned}
$$

Hence, we derive

$$
\left\langle g_{i}\left(y_{i, t}\right)-g_{i}\left(x_{i}\right)+\rho\left[F_{i}\left(x_{1}, x_{2}\right)-\omega_{i}^{*}\right], \eta_{i}\left(v_{i}, z_{i}^{*}\right)\right\rangle_{i}+\rho\left[b_{i}\left(x_{i}, v_{i}\right)-b_{i}\left(x_{i}, z_{i}^{*}\right)\right] \geq 0 .
$$

Let $t \rightarrow 0^{+}$, by the Lipschitz continuity of $g_{i}$, we have

$$
\left\langle g_{i}\left(z_{i}^{*}\right)-g_{i}\left(x_{i}\right)+\rho\left[F_{i}\left(x_{1}, x_{2}\right)-\omega_{i}^{*}\right], \eta_{i}\left(v_{i}, z_{i}^{*}\right)\right\rangle_{i}+\rho\left[b_{i}\left(x_{i}, v_{i}\right)-b_{i}\left(x_{i}, z_{i}^{*}\right)\right] \geq 0 .
$$

Therefore, $\left(z_{1}^{*}, z_{2}^{*}\right)$ is a solution of the auxiliary problems $P\left(x_{1}, x_{2}\right)$.

Now, let $\left(z_{1}^{\prime}, z_{2}^{\prime}\right)$ be another solution of the auxiliary problems $P\left(x_{1}, x_{2}\right)$ which is different from $\left(z_{1}^{*}, z_{2}^{*}\right)$, then we have

$$
\left\langle g_{i}\left(z_{i}^{\prime}\right)-g_{i}\left(x_{i}\right)+\rho\left[F_{i}\left(x_{1}, x_{2}\right)-\omega_{i}^{*}\right], \eta_{i}\left(v_{i}, z_{i}^{\prime}\right)\right\rangle_{i}+\rho\left[b_{i}\left(x_{i}, v_{i}\right)-b_{i}\left(x_{i}, z_{i}^{\prime}\right)\right] \geq 0 .
$$

Taking $v_{i}=z_{i}^{\prime}$ in (2.7) and $v_{i}=z_{i}^{*}$ in (2.8) and adding these two inequalities, we obtain

$$
\left\langle g_{i}\left(z_{i}^{\prime}\right)-g_{i}\left(z_{i}^{*}\right), \eta_{i}\left(z_{i}^{*}, z_{i}^{\prime}\right)\right\rangle_{i} \geq 0 .
$$

Since $g_{i}$ is $\eta_{i}$-strongly monotone, we obtain

$$
\sigma_{i}\left\|z_{i}^{*}-z_{i}^{\prime}\right\|_{i}^{2} \leq\left\langle g_{i}\left(z_{i}^{*}\right)-g_{i}\left(z_{i}^{\prime}\right), \eta_{i}\left(z_{i}^{*}, z_{i}^{\prime}\right)\right\rangle_{i} \leq 0,
$$

and so $\left(z_{1}^{\prime}, z_{2}^{\prime}\right)=\left(z_{1}^{*}, z_{2}^{*}\right)$. This completes the proof.

By virtue of Theorem 2.2, we now construct an iterative algorithm for solving the SGNMVLIP (1.1) and (1.2).

For given $\left(x_{0}, y_{0}\right) \in B_{1} \times B_{2}$, from Theorem 2.2, we know that the auxiliary problems $P\left(x_{0}, y_{0}\right)$ have a solution $\left(x_{1}, y_{1}\right) \in B_{1} \times B_{2}$, that is,

$$
\begin{aligned}
& \left\langle g_{1}\left(x_{1}\right)-g_{1}\left(x_{0}\right)+\rho\left[F_{1}\left(x_{0}, y_{0}\right)-\omega_{1}^{*}\right], \eta_{1}\left(v_{1}, x_{1}\right)\right\rangle_{1}+\rho\left[b_{1}\left(x_{0}, v_{1}\right)-b_{1}\left(x_{0}, x_{1}\right)\right] \geq 0 \quad \forall v_{1} \in B_{1}, \\
& \left\langle g_{2}\left(y_{1}\right)-g_{2}\left(y_{0}\right)+\rho\left[F_{2}\left(x_{0}, y_{0}\right)-\omega_{2}^{*}\right], \eta_{2}\left(v_{2}, y_{1}\right)\right\rangle_{2}+\rho\left[b_{2}\left(y_{0}, v_{2}\right)-b_{2}\left(y_{0}, y_{1}\right)\right] \geq 0 \quad \forall v_{2} \in B_{2} .
\end{aligned}
$$


Journal of Applied Mathematics

Again by Theorem 2.2 , the auxiliary problems $P\left(x_{1}, y_{1}\right)$ have a solution $\left(x_{2}, y_{2}\right) \in B_{1} \times B_{2}$, that is,

$$
\begin{aligned}
& \left\langle g_{1}\left(x_{2}\right)-g_{1}\left(x_{1}\right)+\rho\left[F_{1}\left(x_{1}, y_{1}\right)-\omega_{1}^{*}\right], \eta_{1}\left(v_{1}, x_{2}\right)\right\rangle_{1}+\rho\left[b_{1}\left(x_{1}, v_{1}\right)-b_{1}\left(x_{1}, x_{2}\right)\right] \geq 0 \quad \forall v_{1} \in B_{1}, \\
& \left\langle g_{2}\left(y_{2}\right)-g_{2}\left(y_{1}\right)+\rho\left[F_{2}\left(x_{1}, y_{1}\right)-\omega_{2}^{*}\right], \eta_{2}\left(v_{2}, y_{2}\right)\right\rangle_{2}+\rho\left[b_{2}\left(y_{1}, v_{2}\right)-b_{2}\left(y_{1}, y_{2}\right)\right] \geq 0 \quad \forall v_{2} \in B_{2} .
\end{aligned}
$$

By induction, we can get the iterative algorithm for solving the SGNMVLIP (1.1) and (1.2) as follows.

Algorithm 2.3. For given $\left(x_{0}, y_{0}\right) \in B_{1} \times B_{2}$, there exists a sequence $\left\{\left(x_{n}, y_{n}\right)\right\}$ such that

$$
\begin{array}{r}
\left\langle g_{1}\left(x_{n+1}\right)-g_{1}\left(x_{n}\right)+\rho\left[F_{1}\left(x_{n}, y_{n}\right)-\omega_{1}^{*}\right], \eta_{1}\left(v_{1}, x_{n+1}\right)\right\rangle_{1}+\rho\left[b_{1}\left(x_{n}, v_{1}\right)-b_{1}\left(x_{n}, x_{n+1}\right)\right] \geq 0 \\
\forall v_{1} \in B_{1} \\
\left\langle g_{2}\left(y_{n+1}\right)-g_{2}\left(y_{n}\right)+\rho\left[F_{2}\left(x_{n}, y_{n}\right)-\omega_{2}^{*}\right], \eta_{2}\left(v_{2}, y_{n+1}\right)\right\rangle_{2}+\rho\left[b_{2}\left(y_{n}, v_{2}\right)-b_{2}\left(y_{n}, y_{n+1}\right)\right] \geq 0 \\
\forall v_{2} \in B_{2}, \quad n=0,1,2, \ldots,
\end{array}
$$

where $\rho>0$ is a constant.

\section{Existence of Convergence Theorem}

In this section, we will prove not only that the sequence $\left\{\left(x_{n}, y_{n}\right)\right\}$ generated by Algorithm 2.3 converges strongly to $\left(x^{*}, y^{*}\right)$, and also that $\left(x^{*}, y^{*}\right)$ is a solution of SGNMVLIP (1.1) and (1.2).

Theorem 3.1. For each $i \in I$, assume that the following conditions are satisfied:

(1) $g_{i}: B_{i} \rightarrow B_{i}^{*}$ is $\eta_{i}$-strongly monotone and Lipschtiz continuous with constant $\sigma_{i}>0$ and $\mu_{i}>0$, respectively;

(2) $\eta_{i}: B_{i} \times B_{i} \rightarrow B_{i}$ is Lipschtiz continuous with constant $\delta_{i}>0$;

(3) $F_{i}: B_{1} \times B_{2} \rightarrow B_{i}^{*}$ is $\left(\lambda_{i}, \xi_{i}\right)$-Lipschtiz continuous;

(4) $F_{i}: B_{1} \times B_{2} \rightarrow B_{i}^{*}$ is $\eta_{i}$-strongly monotone in the ith argument with constant $\varepsilon_{i}>0$;

(5) $b_{i}: B_{i} \times B_{i} \rightarrow \mathbb{R}$ satisfies the properties (i)-(iv).

If Assumption 1.4 holds and there exists a constant $\rho>0$ such that

$$
\begin{aligned}
& P_{1}=2 \sigma_{1}-\frac{1}{4 \rho \varepsilon_{1}}\left[\delta_{1}^{2} \mu_{1}^{2}+\rho \delta_{1}^{2} \mu_{1} \lambda_{1}+2 \rho^{2} \varepsilon_{1}\left(\gamma_{1}+\delta_{1} \xi_{1}+2 \delta_{1} \lambda_{1}\right)\right]>0, \\
& P_{2}=2 \sigma_{2}-\frac{1}{4 \rho \varepsilon_{2}}\left[\delta_{2}^{2} \mu_{2}^{2}+\rho \delta_{2}^{2} \mu_{2} \xi_{2}+2 \rho^{2} \varepsilon_{2}\left(\gamma_{2}+\delta_{2} \lambda_{2}+2 \delta_{2} \xi_{2}\right)\right]>0
\end{aligned}
$$




$$
\begin{aligned}
& \frac{1}{4 \varepsilon_{1}}\left[\delta_{1}^{2} \mu_{1} \lambda_{1}+\rho\left(\delta_{1}^{2} \lambda_{1}^{2}+2 \varepsilon_{1} \gamma_{1}\right)\right]+\frac{1}{2} \rho \lambda_{2} \delta_{2}<\min \left\{P_{1}, P_{2}\right\} \\
& \frac{1}{4 \varepsilon_{2}}\left[\delta_{2}^{2} \mu_{2} \xi_{2}+\rho\left(\delta_{2}^{2} \xi_{2}^{2}+2 \varepsilon_{2} \gamma_{2}\right)\right]+\frac{1}{2} \rho \xi_{1} \delta_{1}<\min \left\{P_{1}, P_{2}\right\}
\end{aligned}
$$

then the sequence $\left\{\left(x_{n}, y_{n}\right)\right\}$ generated by Algorithm 2.3 converges strongly to $\left(x^{*}, y^{*}\right)$, and $\left(x^{*}, y^{*}\right)$ is a solution of SGNMVLIP (1.1) and (1.2).

Proof. For any $\left(v_{1}, v_{2}\right) \in B_{1} \times B_{2}$, it follows from Algorithm 2.3 that

$$
\begin{gathered}
\left\langle g_{1}\left(x_{n}\right)-g_{1}\left(x_{n-1}\right)+\rho\left[F_{1}\left(x_{n-1}, y_{n-1}\right)-\omega_{1}^{*}\right], \eta_{1}\left(v_{1}, x_{n}\right)\right\rangle_{1}+\rho\left[b_{1}\left(x_{n-1}, v_{1}\right)-b_{1}\left(x_{n-1}, x_{n}\right)\right] \geq 0, \\
\left\langle g_{2}\left(y_{n}\right)-g_{2}\left(y_{n-1}\right)+\rho\left[F_{2}\left(x_{n-1}, y_{n-1}\right)-\omega_{2}^{*}\right], \eta_{2}\left(v_{2}, y_{n}\right)\right\rangle_{2}+\rho\left[b_{2}\left(y_{n-1}, v_{2}\right)-b_{2}\left(y_{n-1}, y_{n}\right)\right] \geq 0, \\
\left\langle g_{1}\left(x_{n+1}\right)-g_{1}\left(x_{n}\right)+\rho\left[F_{1}\left(x_{n}, y_{n}\right)-\omega_{1}^{*}\right], \eta_{1}\left(v_{1}, x_{n+1}\right)\right\rangle_{1}+\rho\left[b_{1}\left(x_{n}, v_{1}\right)-b_{1}\left(x_{n}, x_{n+1}\right)\right] \geq 0, \\
\left\langle g_{2}\left(y_{n+1}\right)-g_{2}\left(y_{n}\right)+\rho\left[F_{2}\left(x_{n}, y_{n}\right)-\omega_{2}^{*}\right], \eta_{2}\left(v_{2}, y_{n+1}\right)\right\rangle_{2}+\rho\left[b_{2}\left(y_{n}, v_{2}\right)-b_{2}\left(y_{n}, y_{n+1}\right)\right] \geq 0 .
\end{gathered}
$$

Taking $v_{1}=x_{n+1}$ in (3.3) and $v_{1}=x_{n}$ in (3.5), respectively, we get

$$
\begin{gathered}
\left\langle g_{1}\left(x_{n}\right)-g_{1}\left(x_{n-1}\right)+\rho\left[F_{1}\left(x_{n-1}, y_{n-1}\right)-\omega_{1}^{*}\right], \eta_{1}\left(x_{n+1}, x_{n}\right)\right\rangle_{1}+\rho\left[b_{1}\left(x_{n-1}, x_{n+1}\right)-b_{1}\left(x_{n-1}, x_{n}\right)\right] \geq 0, \\
\left\langle g_{1}\left(x_{n+1}\right)-g_{1}\left(x_{n}\right)+\rho\left[F_{1}\left(x_{n}, y_{n}\right)-\omega_{1}^{*}\right], \eta_{1}\left(x_{n}, x_{n+1}\right)\right\rangle_{1}+\rho\left[b_{1}\left(x_{n}, x_{n}\right)-b_{1}\left(x_{n}, x_{n+1}\right)\right] \geq 0 .
\end{gathered}
$$

Adding (3.7) and (3.8), we obtain

$$
\begin{aligned}
\left\langle g_{1}\left(x_{n}\right)\right. & \left.-g_{1}\left(x_{n+1}\right), \eta_{1}\left(x_{n}, x_{n+1}\right)\right\rangle_{1} \\
\leq & \left\langle g_{1}\left(x_{n-1}\right)-g_{1}\left(x_{n}\right)-\rho\left[F_{1}\left(x_{n-1}, y_{n-1}\right)-F_{1}\left(x_{n}, y_{n}\right)\right], \eta_{1}\left(x_{n}, x_{n+1}\right)\right\rangle_{1} \\
& +\rho\left[b_{1}\left(x_{n}-x_{n-1}, x_{n}\right)-b_{1}\left(x_{n}-x_{n-1}, x_{n+1}\right)\right] .
\end{aligned}
$$

From conditions (1) and (2), we have

$$
\begin{aligned}
\left\langle g_{1}\left(x_{n}\right)-g_{1}\left(x_{n+1}\right), \eta_{1}\left(x_{n}, x_{n+1}\right)\right\rangle_{1} \geq & \sigma_{1}\left\|x_{n}-x_{n+1}\right\|_{1}^{2} \\
\left\langle g_{1}\left(x_{n-1}\right)-g_{1}\left(x_{n}\right), \eta_{1}\left(x_{n}, x_{n+1}\right)\right\rangle_{1}= & \left\langle g_{1}\left(x_{n-1}\right)-g_{1}\left(x_{n+1}\right), \eta_{1}\left(x_{n}, x_{n+1}\right)\right\rangle_{1} \\
& +\left\langle g_{1}\left(x_{n+1}\right)-g_{1}\left(x_{n}\right), \eta_{1}\left(x_{n}, x_{n+1}\right)\right\rangle_{1} \\
\leq & \delta_{1} \mu_{1}\left\|x_{n+1}-x_{n-1}\right\|_{1}\left\|x_{n+1}-x_{n}\right\|_{1}-\sigma_{1}\left\|x_{n+1}-x_{n}\right\|_{1}^{2} .
\end{aligned}
$$


Journal of Applied Mathematics

From conditions (3) and (4), we obtain

$$
\begin{aligned}
&\left\langle F_{1}\left(x_{n}, y_{n}\right)-F_{1}\left(x_{n-1}, y_{n-1}\right), \eta_{1}\left(x_{n}, x_{n+1}\right)\right\rangle_{1} \\
&=\left\langle F_{1}\left(x_{n}, y_{n}\right)-F_{1}\left(x_{n+1}, y_{n-1}\right), \eta_{1}\left(x_{n}, x_{n+1}\right)\right\rangle_{1} \\
&+\left\langle F_{1}\left(x_{n+1}, y_{n-1}\right)-F_{1}\left(x_{n-1}, y_{n-1}\right), \eta_{1}\left(x_{n}, x_{n-1}\right)\right\rangle_{1} \\
&+\left\langle F_{1}\left(x_{n+1}, y_{n-1}\right)-F_{1}\left(x_{n-1}, y_{n-1}\right), \eta_{1}\left(x_{n-1}, x_{n+1}\right)\right\rangle_{1} \\
& \leq \delta_{1} \lambda_{1}\left\|x_{n+1}-x_{n}\right\|_{1}^{2}+\delta_{1} \xi_{1}\left\|x_{n+1}-x_{n}\right\| 1\left\|y_{n}-y_{n-1}\right\|_{2} \\
&+\delta_{1} \lambda_{1}\left\|x_{n+1}-x_{n-1}\right\|_{1}\left\|x_{n}-x_{n-1}\right\|_{1}-\varepsilon_{1}\left\|x_{n+1}-x_{n-1}\right\|_{1}^{2} .
\end{aligned}
$$

From condition (5) and Remark 1.1 (2), we have

$$
b_{1}\left(x_{n}-x_{n-1}, x_{n}\right)-b_{1}\left(x_{n}-x_{n-1}, x_{n+1}\right) \leq \gamma_{1}\left\|x_{n+1}-x_{n}\right\|_{1}\left\|x_{n}-x_{n-1}\right\|_{1} .
$$

Therefore, from (3.9)-(3.12), we derive

$$
\begin{aligned}
&\left(2 \sigma_{1}-\rho \delta_{1} \lambda_{1}\right)\left\|x_{n}-x_{n+1}\right\|_{1}^{2} \\
& \leq-\rho \varepsilon_{1}\left\|x_{n+1}-x_{n-1}\right\|_{1}^{2}+\left[\delta_{1} \mu_{1}\left\|x_{n+1}-x_{n}\right\|_{1}+\rho \delta_{1} \lambda_{1}\left\|x_{n}-x_{n-1}\right\|_{1}\right]\left\|x_{n+1}-x_{n-1}\right\|_{1} \\
&+\rho \delta_{1} \xi_{1}\left\|x_{n+1}-x_{n}\right\|_{1}\left\|y_{n}-y_{n-1}\right\|_{2}+\rho \gamma_{1}\left\|x_{n+1}-x_{n}\right\|_{1}\left\|x_{n}-x_{n-1}\right\|_{1} \\
& \leq \frac{1}{4 \rho \varepsilon_{1}}\left[\delta_{1} \mu_{1}\left\|x_{n+1}-x_{n}\right\|_{1}+\rho \delta_{1} \lambda_{1}\left\|x_{n}-x_{n-1}\right\|_{1}\right]^{2} \\
&+\rho \delta_{1} \xi_{1}\left\|x_{n+1}-x_{n}\right\|_{1}\left\|y_{n}-y_{n-1}\right\|_{2}+\rho \gamma_{1}\left\|x_{n+1}-x_{n}\right\|_{1}\left\|x_{n}-x_{n-1}\right\|_{1} \\
&= \frac{\delta_{1}^{2} \mu_{1}^{2}}{4 \rho \varepsilon_{1}}\left\|x_{n+1}-x_{n}\right\|_{1}^{2}+\frac{\rho \delta_{1}^{2} \lambda_{1}^{2}}{4 \varepsilon_{1}}\left\|x_{n}-x_{n-1}\right\|_{1}^{2} \\
&+\left[\frac{\delta_{1}^{2} \mu_{1} \lambda_{1}}{2 \varepsilon_{1}}+\rho \gamma_{1}\right]\left\|x_{n+1}-x_{n}\right\|_{1}\left\|x_{n}-x_{n-1}\right\|_{1}+\rho \delta_{1} \xi_{1}\left\|x_{n+1}-x_{n}\right\|_{1}\left\|y_{n}-y_{n-1}\right\|_{2} \\
& \leq \frac{1}{4 \rho \varepsilon_{1}}\left[\delta_{1}^{2} \mu_{1}^{2}+\rho \delta_{1}^{2} \mu_{1} \lambda_{1}+2 \rho^{2} \varepsilon_{1}\left(\gamma_{1}+\delta_{1} \xi_{1}\right)\right]\left\|x_{n+1}-x_{n}\right\|_{1}^{2} \\
&+\frac{1}{4 \varepsilon_{1}}\left[\delta_{1}^{2} \mu_{1} \lambda_{1}+\rho\left(\delta_{1}^{2} \lambda_{1}^{2}+2 \varepsilon_{1} \gamma_{1}\right)\right]\left\|x_{n}-x_{n-1}\right\|_{1}^{2}+\frac{1}{2} \rho \delta_{1} \xi_{1}\left\|y_{n}-y_{n-1}\right\|_{2}^{2}
\end{aligned}
$$

which implies

$$
P_{1}\left\|x_{n}-x_{n+1}\right\|_{1}^{2} \leq Q_{1}\left\|x_{n}-x_{n-1}\right\|_{1}^{2}+R_{1}\left\|y_{n}-y_{n-1}\right\|_{2}^{2}
$$

where $Q_{1}=\left(1 / 4 \varepsilon_{1}\right)\left[\delta_{1}^{2} \mu_{1} \lambda_{1}+\rho\left(\delta_{1}^{2} \lambda_{1}^{2}+2 \varepsilon_{1} \gamma_{1}\right)\right], R_{1}=(1 / 2) \rho \delta_{1} \xi_{1}$. 
Taking $v_{2}=y_{n+1}$ in (3.4) and $v_{2}=y_{n}$ in (3.6), respectively, we get

$$
\begin{gathered}
\left\langle g_{2}\left(y_{n}\right)-g_{2}\left(y_{n-1}\right)+\rho\left[F_{2}\left(x_{n-1}, y_{n-1}\right)-\omega_{2}^{*}\right], \eta_{2}\left(y_{n+1}, y_{n}\right)\right\rangle_{2}+\rho\left[b_{2}\left(y_{n-1}, y_{n+1}\right)-b_{2}\left(y_{n-1}, y_{n}\right)\right] \geq 0, \\
\left\langle g_{2}\left(y_{n+1}\right)-g_{2}\left(y_{n}\right)+\rho\left[F_{2}\left(x_{n}, y_{n}\right)-\omega_{2}^{*}\right], \eta_{2}\left(y_{n}, y_{n+1}\right)\right\rangle_{2}+\rho\left[b_{2}\left(y_{n}, y_{n}\right)-b_{2}\left(y_{n}, y_{n+1}\right)\right] \geq 0 .
\end{gathered}
$$

Adding (3.15), we obtain

$$
\begin{aligned}
\left\langle g_{2}\left(y_{n}\right)\right. & \left.-g_{2}\left(y_{n+1}\right), \eta_{2}\left(y_{n}, y_{n+1}\right)\right\rangle_{2} \\
\leq & \left\langle g_{2}\left(y_{n-1}\right)-g_{2}\left(y_{n}\right)-\rho\left[F_{2}\left(x_{n-1}, y_{n-1}\right)-F_{2}\left(x_{n}, y_{n}\right)\right], \eta_{2}\left(y_{n}, y_{n+1}\right)\right\rangle_{2} \\
& +\rho\left[b_{2}\left(y_{n}-y_{n-1}, y_{n}\right)-b_{2}\left(y_{n}-y_{n-1}, y_{n+1}\right)\right] .
\end{aligned}
$$

From conditions (1) and (2), we have

$$
\begin{aligned}
\left\langle g_{2}\left(y_{n}\right)-g_{2}\left(y_{n+1}\right), \eta_{2}\left(y_{n}, y_{n+1}\right)\right\rangle_{2} \geq & \sigma_{2}\left\|y_{n}-y_{n+1}\right\|_{2^{\prime}}^{2} \\
\left\langle g_{2}\left(y_{n-1}\right)-g_{2}\left(y_{n}\right), \eta_{2}\left(y_{n}, y_{n+1}\right)\right\rangle_{2}= & \left\langle g_{2}\left(y_{n-1}\right)-g_{2}\left(y_{n+1}\right), \eta_{2}\left(y_{n}, y_{n+1}\right)\right\rangle_{2} \\
& +\left\langle g_{2}\left(y_{n+1}\right)-g_{2}\left(y_{n}\right), \eta_{2}\left(y_{n}, y_{n+1}\right)\right\rangle_{2} \\
\leq & \delta_{2} \mu_{2}\left\|y_{n+1}-y_{n-1}\right\|\left\|_{2}\right\| y_{n+1}-y_{n}\left\|_{2}-\sigma_{2}\right\| y_{n+1}-y_{n} \|_{2}^{2} .
\end{aligned}
$$

From conditions (3) and (4), we obtain

$$
\begin{aligned}
&\left\langle F_{2}\left(x_{n}, y_{n}\right)-F_{2}\left(x_{n-1}, y_{n-1}\right), \eta_{2}\left(y_{n}, y_{n+1}\right)\right\rangle_{2} \\
&=\left\langle F_{2}\left(x_{n}, y_{n}\right)-F_{2}\left(x_{n-1}, y_{n+1}\right), \eta_{2}\left(y_{n}, y_{n+1}\right)\right\rangle_{2} \\
&+\left\langle F_{2}\left(x_{n-1}, y_{n+1}\right)-F_{2}\left(x_{n-1}, y_{n-1}\right), \eta_{2}\left(y_{n}, y_{n-1}\right)\right\rangle_{2} \\
&+\left\langle F_{2}\left(x_{n-1}, y_{n+1}\right)-F_{2}\left(x_{n-1}, y_{n-1}\right), \eta_{2}\left(y_{n-1}, y_{n+1}\right)\right\rangle_{2} \\
& \leq \delta_{2} \xi_{2}\left\|y_{n+1}-y_{n}\right\|_{2}^{2}+\delta_{2} \lambda_{2}\left\|y_{n+1}-y_{n}\right\|_{2}\left\|x_{n}-x_{n-1}\right\|_{1} \\
&+\delta_{2} \xi_{2}\left\|y_{n+1}-y_{n-1}\right\|_{2}\left\|y_{n}-y_{n-1}\right\|_{2}-\varepsilon_{2}\left\|y_{n+1}-y_{n-1}\right\|_{2}^{2}
\end{aligned}
$$

From condition (5) and Remark 1.1 (2), we have

$$
b_{2}\left(y_{n}-y_{n-1}, y_{n}\right)-b_{2}\left(y_{n}-y_{n-1}, y_{n+1}\right) \leq \gamma_{2}\left\|y_{n+1}-y_{n}\right\|_{2}\left\|y_{n}-y_{n-1}\right\|_{2} .
$$


Therefore, from (3.16)-(3.19), we derive

$$
\begin{aligned}
\left(2 \sigma_{2}-\right. & \left.\rho \delta_{2} \xi_{2}\right)\left\|y_{n}-y_{n+1}\right\|_{2}^{2} \\
\leq & -\rho \varepsilon_{2}\left\|y_{n+1}-y_{n-1}\right\|_{2}^{2}+\left[\delta_{2} \mu_{2}\left\|y_{n+1}-y_{n}\right\|_{2}+\rho \delta_{2} \xi_{2}\left\|y_{n}-y_{n-1}\right\|_{2}\right]\left\|y_{n+1}-y_{n-1}\right\|_{2} \\
& +\rho \delta_{2} \lambda_{2}\left\|y_{n+1}-y_{n}\right\|_{2}\left\|x_{n}-x_{n-1}\right\|_{1}+\rho \gamma_{2}\left\|y_{n+1}-y_{n}\right\|_{2}\left\|y_{n}-y_{n-1}\right\|_{2} \\
\leq & \frac{1}{4 \rho \varepsilon_{2}}\left[\delta_{2} \mu_{2}\left\|y_{n+1}-y_{n}\right\|_{2}+\rho \delta_{2} \xi_{2}\left\|y_{n}-y_{n-1}\right\|_{2}\right]^{2} \\
& +\rho \delta_{2} \lambda_{2}\left\|y_{n+1}-y_{n}\right\|_{2}\left\|x_{n}-x_{n-1}\right\|_{1}+\rho \gamma_{2}\left\|y_{n+1}-y_{n}\right\|_{2}\left\|y_{n}-y_{n-1}\right\|_{2} \\
= & \frac{\delta_{2}^{2} \mu_{2}^{2}}{4 \rho \varepsilon_{2}}\left\|y_{n+1}-y_{n}\right\|_{2}^{2}+\frac{\rho \delta_{2}^{2} \xi_{2}^{2}}{4 \varepsilon_{2}}\left\|y_{n}-y_{n-1}\right\|_{2}^{2} \\
& +\left[\frac{\delta_{2}^{2} \mu_{2} \xi_{2}}{2 \varepsilon_{2}}+\rho \gamma_{2}\right]\left\|y_{n+1}-y_{n}\right\|_{2}\left\|y_{n}-y_{n-1}\right\|_{2}+\rho \delta_{2} \lambda_{2}\left\|y_{n+1}-y_{n}\right\|_{2}\left\|x_{n}-x_{n-1}\right\|_{1} \\
\leq & \frac{1}{4 \rho \varepsilon_{2}}\left[\delta_{2}^{2} \mu_{2}^{2}+\rho \delta_{2}^{2} \mu_{2} \xi_{2}+2 \rho^{2} \varepsilon_{2}\left(\gamma_{2}+\delta_{2} \lambda_{2}\right)\right]\left\|y_{n+1}-y_{n}\right\|_{2}^{2} \\
& +\frac{1}{4 \varepsilon_{2}}\left[\delta_{2}^{2} \mu_{2} \xi_{2}+\rho\left(\delta_{2}^{2} \xi_{2}^{2}+2 \varepsilon_{2} \gamma_{2}\right)\right]\left\|y_{n}-y_{n-1}\right\|_{2}^{2}+\frac{1}{2} \rho \delta_{2} \lambda_{2}\left\|x_{n}-x_{n-1}\right\|_{1}^{2}
\end{aligned}
$$

which implies

$$
P_{2}\left\|y_{n}-y_{n+1}\right\|_{2}^{2} \leq Q_{2}\left\|y_{n}-y_{n-1}\right\|_{2}^{2}+R_{2}\left\|x_{n}-x_{n-1}\right\|_{1}^{2}
$$

where $Q_{2}=\left(1 / 4 \varepsilon_{2}\right)\left[\delta_{2}^{2} \mu_{2} \xi_{2}+\rho\left(\delta_{2}^{2} \xi_{2}^{2}+2 \varepsilon_{2} \gamma_{2}\right)\right], R_{2}=(1 / 2) \rho \delta_{2} \lambda_{2}$.

Adding (3.14) and (3.21), we have

$$
P_{1}\left\|x_{n}-x_{n+1}\right\|_{1}^{2}+P_{2}\left\|y_{n}-y_{n+1}\right\|_{2}^{2} \leq\left(Q_{1}+R_{2}\right)\left\|x_{n}-x_{n-1}\right\|_{1}^{2}+\left(Q_{2}+R_{1}\right)\left\|y_{n}-y_{n-1}\right\|_{2}^{2} .
$$

Define the norm $\|\cdot\|_{*}$ on $B_{1} \times B_{2}$ by

$$
\|(u, v)\|_{*}=\sqrt{\|u\|_{1}^{2}+\|v\|_{2}^{2}} \quad \forall(u, v) \in B_{1} \times B_{2}
$$

it is easy to prove that $\left(B_{1} \times B_{2},\|\cdot\|_{*}\right)$ is a Banach space.

From (3.22), by conditions (3.1), we have

$$
\left\|\left(x_{n+1}, y_{n+1}\right)-\left(x_{n}, y_{n}\right)\right\|_{*}^{2} \leq \max \left\{\theta_{1}, \theta_{2}\right\}\left\|\left(x_{n}, y_{n}\right)-\left(x_{n-1}, y_{n-1}\right)\right\|_{*^{\prime}}^{2}
$$

where $\theta_{1}=\left(Q_{1}+R_{2}\right) / \min \left\{P_{1}, P_{2}\right\}, \theta_{2}=\left(Q_{2}+R_{1}\right) / \min \left\{P_{1}, P_{2}\right\}$. From condition (3.2), which implies $\theta_{1}, \theta_{2} \in(0,1)$, hence $\left\{\left(x_{n}, y_{n}\right)\right\}$ is a cauchy sequence, let $\left(x_{n}, y_{n}\right) \rightarrow\left(x^{*}, y^{*}\right)(n \rightarrow \infty)$. 
By the Lipschitz continuities of $\eta_{1}$ and $g$, and $x_{n} \rightarrow x^{*}(n \rightarrow \infty)$, we have

$$
\left|\left\langle g_{1}\left(x_{n+1}\right)-g_{1}\left(x_{n}\right), \eta_{1}\left(v_{1}, x_{n+1}\right)\right\rangle_{1}\right| \leq \delta_{1} \mu_{1}\left\|x_{n+1}-x_{n}\right\|_{1}\left\|v_{1}-x_{n+1}\right\|_{1} \longrightarrow 0 \quad \text { as } n \longrightarrow \infty \text {. }
$$

Since $F_{1}$ is $\left(\lambda_{1}, \xi_{1}\right)$-Lipschitz, $x_{n} \rightarrow x^{*}(n \rightarrow \infty)$ and $y_{n} \rightarrow y^{*}(n \rightarrow \infty)$, then we obtain

$$
\begin{aligned}
\left|\left\langle F_{1}\left(x_{n}, y_{n}\right)-\omega_{1}^{*}, \eta_{1}\left(v_{1}, x_{n+1}\right)\right\rangle_{1}-\left\langle F_{1}\left(x^{*}, y^{*}\right)-\omega_{1}^{*}, \eta_{1}\left(v_{1}, x^{*}\right)\right\rangle_{1}\right| \\
\leq \mid \\
\leq\left|\left\langle F_{1}\left(x_{n}, y_{n}\right)-F_{1}\left(x^{*}, y^{*}\right), \eta_{1}\left(v_{1}, x_{n+1}\right)\right\rangle_{1}\right|+\left|\left\langle F_{1}\left(x^{*}, y^{*}\right)-\omega_{1}^{*}, \eta_{1}\left(v_{1}, x_{n+1}\right)-\eta_{1}\left(v_{1}, x^{*}\right)\right\rangle_{1}\right| \\
\leq \delta_{1}\left(\lambda_{1}\left\|x_{n}-x^{*}\right\|_{1}+\xi_{1}\left\|y_{n}-y^{*}\right\|_{2}\right)\left\|v_{1}-x_{n+1}\right\|_{1} \\
\quad+\delta_{1}\left\|F_{1}\left(x^{*}, y^{*}\right)-\omega_{1}^{*}\right\|_{1}\left\|x_{n+1}-x^{*}\right\|_{1} \longrightarrow 0 \text { as } n \longrightarrow \infty .
\end{aligned}
$$

From condition (5) and Remark 1.1 (2), we have

$$
\begin{aligned}
\left|b_{1}\left(x_{n}, x_{n+1}\right)-b_{1}\left(x^{*}, x^{*}\right)\right| & \leq\left|b_{1}\left(x_{n}, x_{n+1}\right)-b_{1}\left(x_{n}, x^{*}\right)\right|+\left|b_{1}\left(x_{n}, x^{*}\right)-b_{1}\left(x^{*}, x^{*}\right)\right| \\
& \leq \gamma_{1}\left\|x_{n}\right\|_{1}\left\|x_{n+1}-x^{*}\right\|_{1}+\left|b_{1}\left(x_{n}-x^{*}, x^{*}\right)\right| \\
& \leq \gamma_{1}\left\|x_{n}\right\|_{1}\left\|x_{n+1}-x^{*}\right\|_{1}+\gamma_{1}\left\|x_{n}-x^{*}\right\|_{1}\left\|x^{*}\right\|_{1} \longrightarrow 0 \quad \text { as } n \longrightarrow \infty
\end{aligned}
$$

Hence, as $n \rightarrow \infty$ in (2.13), we obtain

$$
\left\langle F_{1}\left(x^{*}, y^{*}\right)-\omega_{1}^{*}, \eta_{1}\left(v_{1}, x^{*}\right)\right\rangle_{1}+b_{1}\left(x^{*}, v_{1}\right)-b_{1}\left(x^{*}, x^{*}\right) \geq 0 \quad \forall v_{1} \in B_{1} .
$$

It is similar as above, we can obtain

$$
\left\langle F_{2}\left(x^{*}, y^{*}\right)-\omega_{1}^{*}, \eta_{2}\left(v_{2}, y^{*}\right)\right\rangle_{2}+b_{2}\left(y^{*}, v_{2}\right)-b_{2}\left(y^{*}, y^{*}\right) \geq 0 \quad \forall v_{2} \in B_{2}
$$

Therefore, $\left(x^{*}, y^{*}\right)$ is a solution of SGNMLVIP (1.1) and (1.2).

Example 3.2. Let $B_{1}=B_{2}=L^{2}[0,1]=\{x:[0,1] \rightarrow \mathbb{R} \mid x$ be Lebesgue measurable and $\left.\int_{0}^{1} x^{2}(t) d t<+\infty\right\}$, then the dual space $B_{1}^{*}=B_{2}^{*}=L^{2}[0,1]$. For each $x \in B_{i},(x, y) \in B_{1} \times B_{2}$, let the norm $\|x\|=\left(\int_{0}^{1} x^{2}(t) d t\right)^{1 / 2}$, and the inner product $\langle x, y\rangle=\int_{0}^{1} x(t) y(t) d t$.

For each $i \in I$, let the mappings $F_{i}: B_{1} \times B_{2} \rightarrow B_{i}^{*}, \eta_{i}: B_{i} \times B_{i} \rightarrow B_{i}, b_{i}: B_{i} \times B_{i} \rightarrow \mathbb{R}$, $g_{i}: B_{i} \rightarrow B_{i}^{*}$, be defined as for any $(x, y) \in B_{1} \times B_{2},\left(x^{\prime}, y^{\prime}\right) \in B_{i} \times B_{i}, z \in B_{i}$,

$$
\begin{gathered}
F_{1}(x, y)=19 x+\arctan x-y, \quad F_{2}(x, y)=19 y+\arctan y-x \\
\eta_{i}\left(x^{\prime}, y^{\prime}\right)=\frac{9}{10}\left(x^{\prime}-y^{\prime}\right) \\
b_{i}\left(x^{\prime}, y^{\prime}\right)=\frac{1}{5}\left\langle x^{\prime}, y^{\prime}\right\rangle, \quad g_{i}(z)=\frac{1}{20}(19 z+\arctan z)
\end{gathered}
$$


respectively, then we have

(1) $F_{1}$ is Lipschitz continuous with constant $\left(\lambda_{1}, \xi_{1}\right)=(20,1)$ and $\eta_{1}$-strongly monotone in the first argument with constant $\varepsilon_{1}=18$;

(2) $F_{2}$ is Lipschitz continuous with constant $\left(\lambda_{2}, \xi_{2}\right)=(1,20)$ and $\eta_{2}$-strongly monotone in the second argument with constant $\varepsilon_{2}=18$;

(3) for each $i \in I, \eta_{i}$ is Lipschitz continuous with constant $\delta_{i}=9 / 10$;

(4) for each $i \in I, b_{i}$ satisfy properties (i)-(iv) with constant $\gamma_{i}=1 / 5$;

(5) for each $i \in I, g_{i}$ is Lipschitz continuous with constant $\mu_{i}=1$ and $\eta_{i}$-strongly monotone with constant $\sigma_{i}=9 / 10$.

After simple calculations, conditions (3.1) and (3.2) imply that $\rho \in(0.01,0.047)$.

Remark 3.3. Example 3.2 shows that the constant $\rho$ which satisfies the conditions (3.1) and (3.2) can be obtained.

\section{Acknowledgment}

This work is partially supported by the National Natural Science Foundation of China Grant (10771050).

\section{References}

[1] G. Stampacchia, "Formes bilinéaires coercitives sur les ensembles convexes," Les Comptes Rendus de l'Académie des Sciences, vol. 258, pp. 4413-4416, 1964.

[2] Y. Yao, M. A. Noor, and Y.-C. Liou, "Strong convergence of a modified extra-gradient method to the minimum-norm solution of variational inequalities," Abstract and Applied Analysis, vol. 2012, Article ID 817436, 9 pages, 2012.

[3] L. C. Zeng, S. Schaible, and J.-C. Yao, "Iterative algorithm for generalized set-valued strongly nonlinear mixed variational-like inequalities," Journal of Optimation Theory and Applications, vol. 124, no. 3, pp. 725-738, 2005.

[4] Y. Yao, M. A. Noor, and Y.-C. Liou, "Iterative algorithms for general multi-valued variational inequalities," Abstract and Applied Analysis, vol. 2012, Article ID 768272, 10 pages, 2012.

[5] Y. Yao, M. A. Noor, and Y.-C. Liou, "Hierarchical convergence of an implicit double-net algorithm for nonexpansive semigroups and variational inequalities," Fixed Point Theory and Applications, vol. 2011, p. 101, 2011.

[6] M. A. Noor, "Mixed variational-like inequalities," Communications on Applied Nonlinear Analysis, vol. 1, no. 4, pp. 63-75, 1994.

[7] M. A. Noor, "Iterative schemes for multivalued quasi variational inclusions," Journal of Global Optimization, vol. 19, no. 2, pp. 141-150, 2001.

[8] X. H. Chen and Y. F. Liu, "A generalized nonlinear variational-like inequality in reflexive Banach spaces," Journal of Nanjing University Mathematic Biquart, vol. 18, no. 1, pp. 96-103, 2001.

[9] J. Parida, M. Sahoo, and A. Kumar, "A variational-like inequality problem," Bulletin of the Australian Mathematical Society, vol. 39, no. 2, pp. 225-231, 1989.

[10] R. Glowinski, J.-L. Lions, and R. Tremolieres, Numerical Analysis of Variational Inequalities, North-Holland Publishing, New York, NY, USA, 1981.

[11] J. Shen and L.-P. Pang, "A bundle-type auxiliary problem method for solving generalized variationallike inequalities," Computers \& Mathematics with Applications, vol. 55, no. 12, pp. 2993-2998, 2008.

[12] J. Y. Park and J. U. Jeong, "Generalized nonlinear variational-like inequalities in reflexive Banach spaces," Mathematical Methods in the Applied Sciences, vol. 33, no. 13, pp. 1637-1646, 2010.

[13] M. A. Noor, "Auxiliary principle for generalized mixed variational-like inequalities," Journal of Mathematical Analysis and Applications, vol. 215, no. 1, pp. 75-85, 1997. 
[14] L.-C. Ceng, S.-M. Guu, and J.-C. Yao, "Iterative algorithm for finding approximate solutions of mixed quasi-variational-like inclusions," Computers $\mathcal{E}$ Mathematics with Applications, vol. 56, no. 4, pp. $942-$ 952, 2008.

[15] X. P. Ding, J.-C. Yao, and L.-C. Zeng, “Existence and algorithm of solutions for generalized strongly nonlinear mixed variational-like inequalities in Banach spaces," Computers \& Mathematics with Applications, vol. 55, no. 4, pp. 669-679, 2008.

[16] K. R. Kazmi and F. A. Khan, "Auxiliary problems and algorithm for a system of generalized variational-like inequality problems," Applied Mathematics and Computation, vol. 187, no. 2, pp. 789-796, 2007.

[17] X. P. Ding, "General algorithm for nonlinear variational-like inequalities in reflexive Banach spaces," Indian Journal of Pure and Applied Mathematics, vol. 29, no. 2, pp. 109-120, 1998.

[18] C. E. Chidume, K. R. Kazmi, and H. Zegeye, "General auxiliary problem and algorithm for a generalized multi-valued variational-like inequality problem in reflexive Banach spaces," Applicable Analysis. An International Journal, vol. 82, no. 12, pp. 1099-1109, 2003.

[19] Y. Yao, Y. -C. Liou, and S. M. Kang, "Two-step projection methods for a system of variational inequality problems in Banach spaces," Journal of Global Optimization. In press.

[20] S. S. Chang and S. W. Xiang, "Existence of solutions for a class of quasibilinear variational inequalities," Journal of Systems Science and Mathematical Sciences, vol. 16, no. 2, pp. 136-140, 1996. 


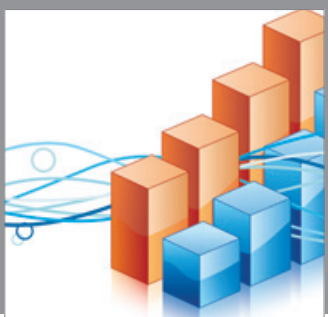

Advances in

Operations Research

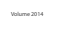

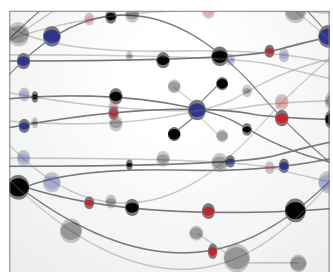

\section{The Scientific} World Journal
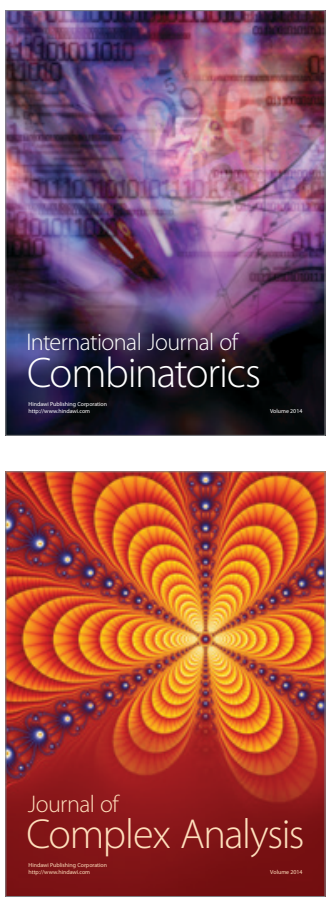

International Journal of

Mathematics and

Mathematical

Sciences
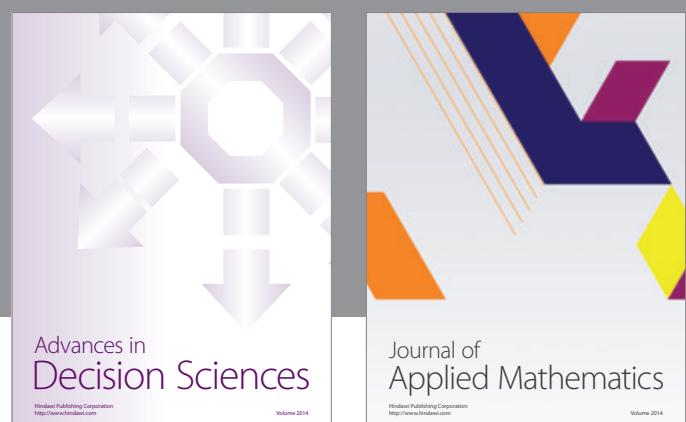

Journal of

Applied Mathematics
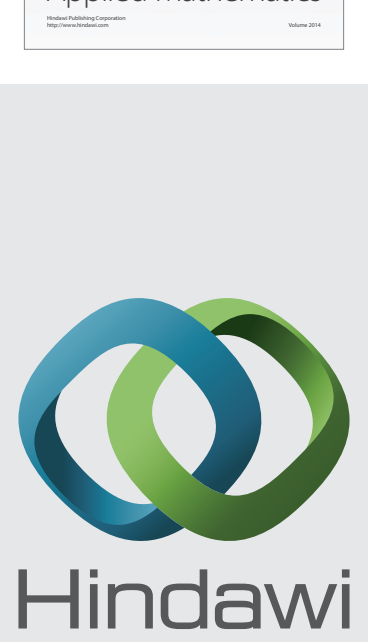

Submit your manuscripts at http://www.hindawi.com
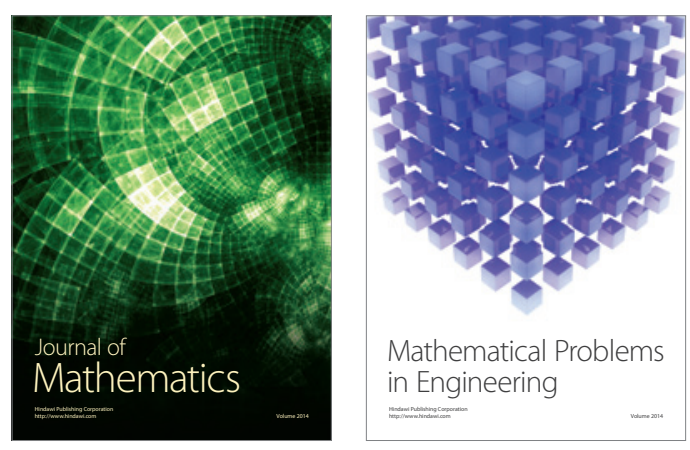

Mathematical Problems in Engineering
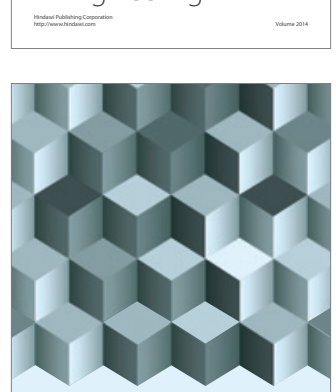

Journal of

Function Spaces
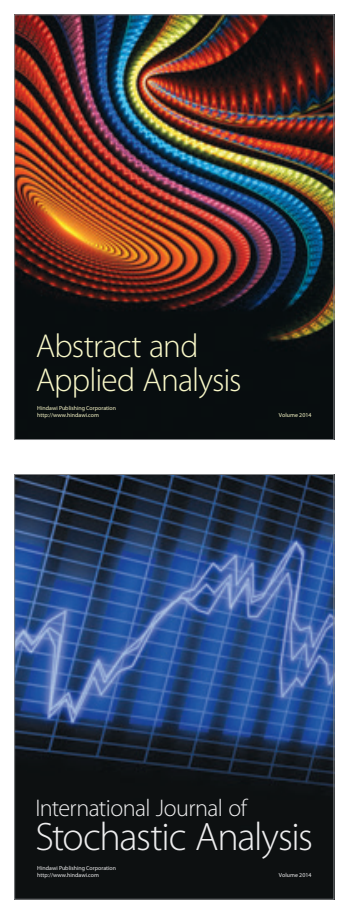

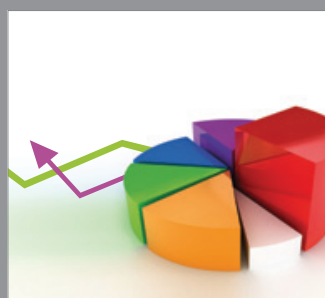

ournal of

Probability and Statistics

Promensencen
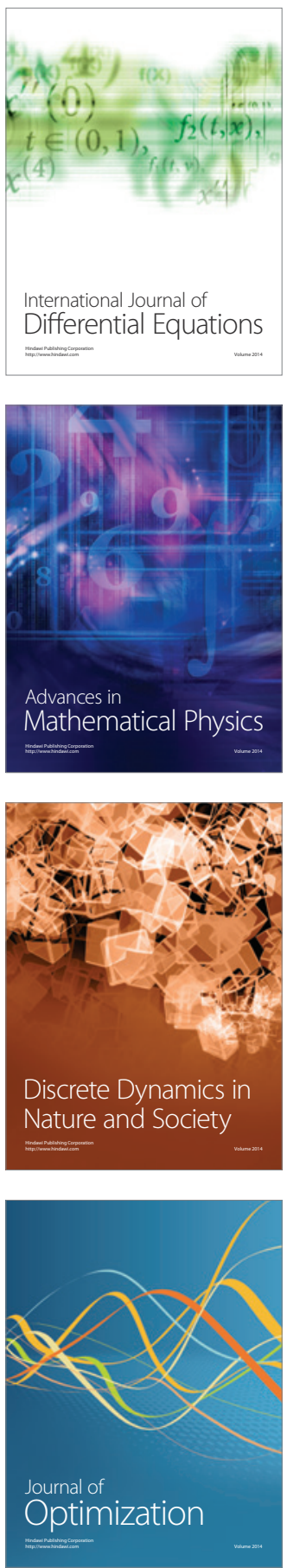